Voix et Images

volxetimages

\title{
Un héros semi-sartrien : Mathieu, de Françoise Loranger
}

\section{André Brochu}

Volume 20, numéro 1 (58), automne 1994

Saint-Denys Garneau

URI : https://id.erudit.org/iderudit/201146ar

DOI : https://doi.org/10.7202/201146ar

Aller au sommaire du numéro

Éditeur(s)

Université du Québec à Montréal

ISSN

0318-9201 (imprimé)

1705-933X (numérique)

Découvrir la revue

Citer cet article

Brochu, A. (1994). Un héros semi-sartrien : Mathieu, de Françoise Loranger.

Voix et Images, 20(1), 178-189. https://doi.org/10.7202/201146ar d'utilisation que vous pouvez consulter en ligne.

https://apropos.erudit.org/fr/usagers/politique-dutilisation/ 


\title{
Un héros semi-sartrien: Mathieu, de Françoise Loranger ${ }^{1}$
}

\author{
André Brochu, Université de Montréal
}

\section{L'athéisme avant la Révolution tranquille}

Dans Un ange cornu avec des ailes de tôle, Michel Tremblay raconte sa découverte de Bonbeur d'occasion, roman qui prendra pour lui une importance considérable, comme c'était le cas pour' la grosse femme dans les Chroniques du plateau Mont-Royal. C'était "un livre athée", écrit-il, "comme certains de ces romans français que lisait ma mère presque en cachette... ${ }^{2}$ "Et il énumère les raisons d'un tel jugement: "Rose-Anna Lacasse qui donnait naissance à un bébé le jour du mariage de sa fille tout en s'abîmant dans la douleur de la perte prochaine de son Daniel, huit ans, qui se mourait lentement de leucémie; Florentine qui épousait Emmanuel Létourneau sans lui dire qu'elle ne pourrait jamais l'aimer et qu'elle était enceinte de Jean Lévesque; Azarius Lacasse qui, à trente-neuf ans, entrait dans l'armée pour que sa famille puisse manger, et je me disais: "C'est ça, la vie, la vraie vie, y'a pas d'explications à l'injustice ni de solution! Le bon Dieu va pas apparaître comme Superman pour sauver tout le monde, ces personnages-là sont perdus 3 ! " Jugement fort intéressant et d'une grande lucidité parce que, même si Gabrielle Roy n'est pas athée, le monde qu'elle décrit est essentiellement compatible, lui, avec l'athéisme ou le désespoir, c'est-à-dire l'absence de foi dans un salut. La misère, la guerre, la souffrance n'ont pas de sens, ne préparent pas les êtres pour une vie future, une autre vie qui permettrait une revanche sur la vie présente. Cette façon de voir les choses, qui récuse les illusions de la foi ou de l'idéalisme, est le fondement même de la modernité, même si elle se présente sous des dehors traditionnels qui sont ceux, par exemple en ce qui concerne Bonbeur d'occasion, du grand roman réaliste ${ }^{4}$ tel qu'il s'est formé au xIx ${ }^{e}$ siècle. Ce roman construit une représentation du vécu très poussée, à la fois dans le sens de la quotidienneté (on voit agir et exister les êtres de très près, dans tout le détail de leurs comportements) et dans la dimension sociale (les êtres nous sont montrés dans les rapports qui les lient aux 
autres de leur classe et aux êtres de classes différentes). Cette représentation du vécu n'est pas sacralisante, elle est plus ou moins explicitement critique et montre la réalité dans ses aspects souvent les plus noirs, étant bien entendu que les gens heureux n'ont pas d'histoire, donc ne peuvent être les sujets d'une action romanesque, et que la littérature, à une époque où sévissent la guerre, la pauvreté, le chômage et la violence sous toutes ses formes, peut difficilement éviter, si elle veut parler du réel, de les représenter. Le roman, en 1945, au Québec comme ailleurs, grâce à Gabrielle Roy, c'est donc cette image "athée " d'un monde voué au malheur.

Et en 1949, une autre femme écrivain, Françoise Loranger, qui est née en 1916 (sept ans après Gabrielle Roy, quatre ans après SaintDenys Garneau et l'année même de la naissance d'Anne Hébert), qui appartient à la génération dés écrivains de La Relève, va elle aussi donner une représentation athée du monde, donc une représentation parallèle à celle de Gabrielle Roy, mais assez différente sur bon nombre de points. Bonbeur d'occasion est un roman fortement social; Matbieu ${ }^{5}$ - comme son titre le suggère déjà - est un roman plutôt psychologique, centré sur un individu (et l'individualité est le domaine d'élection de la psychologie). Les réalités sociales ne sont pas absentes pour autant, mais elles sont subordonnées à celles-là.

Le roman psychologique, qui peint des êtres de la ville et qui s'attache surtout à leur intériorité, constitue un pas de plus sur la voie de la modernité, s'il est vrai, comme l'affirment certains philosophes et anthropologues, que les temps modernes voient le triomphe de l'homo æqualis qui est l'individu égalitaire des sociétés démocratiques, alors que la tradition est le temps de l'bomo bierarchicus et du holisme, qui dissout l'individu dans la totalité ${ }^{6}$. Chez Gabrielle Roy, la société est en train de se défaire, et particulièrement la famille, soumise à d'importantes forces de dislocation. Mais on n'est pas encore installé dans le régime de l'individuel, comme c'est le cas dans Matbieu qui, du reste, peint un milieu beaucoup plus favorisé. Certes, dans Mathieu, la famille existe encore, mais elle est le lieu de tensions terribles, et c'est l'individu qui fait la loi. La famille est au service de l'individu, et non l'inverse (comme, par exemple, dans la société paysanne).

Pour Mathieu, la famille aussi bien que la société sont vécues comme les occasions d'un supplice constamment renouvelé. Le jeune homme est un écorché vif, tout le blesse et il est lui-même l'artisan principal de sa souffrance. Le malheur qu'il vit est sans rémission. Roman athée donc, même si l'auteur ne l'est pas (d'ailleurs le livre contient des passages assez édifiants, mais qui n'enlèvent rien à 
l'authenticité du désespoir peint dans la majeure partie du livre). Le salut de Mathieu - car le personnage a ceci d'original que, plongé depuis toujours au plus profond du gouffre, il finit tout de même par se tirer d'affaire complètement, sans laisser un morceau de corps ou d'âme derrière lui - n'est pas un salut tombé d'en haut, effet d'une grâce impérieuse qui serait la manifestation d'une transcendance, mais un salut monté du corps, des muscles, de la chair, de la vie brute qui est antérieure aux mots.et aux maux.

\section{Mathieu et son entourage}

L'attitude de Mathieu face aux autres et à lui-même nous est donnée dès le premier chapitre - d'ailleurs, dès qu'ils apparaissent, les personnages de Loranger sont dessinés d'un trait sûr, caractérisés avec une grande netteté, de sorte qu'ils évoluent sous nos yeux comme des personnages de théâtre. On sait que Françoise Loranger est avant tout une dramaturge, et cela se sent dès son premier livre. Le personnage dramatique, même s'il met du temps à révéler son intériorité, est tout de même d'emblée une présence complète sur la scène; son apparence physique, ses gestes, son comportement imposent au spectateur une entité bien délimitée. C'est l'équivalent de cette présence que la romancière cherche à créer par les croquis rapides et définitifs qui nous livrent d'emblée l'essentiel des personnages.

Mathieu nous apparaît comme un être hyperlucide, au milieu de gens qui semblent vivre presque tous à la surface d'eux-mêmes, pris dans des rôles qui les détournent de leur propre vérité.

Ce sentiment d'être en retrait par rapport aux autres, et d'être le seul à percevoir la réalité des choses, la profondeur du réel, alors que les autres se leurrent, se cachent à eux-mêmes ce qu'ils sont et ce qu'ils font, se jouent la comédie à eux-mêmes et à leur entourage, se " font des accroires" en ce qui concerne le sens de l'existence, s'illusionnent sur la réalité de Dieu, des principes, des valeurs, des lois, voilà une attitude tout à fait d'époque en littérature. On en trouve l'affirmation très brillante en France chez un Sartre (La Nausée, Les Chemins de la libertê) et un Camus (L'Étranger). Pour Sartre, l'homme est une liberté jetée dans le monde, et la liberté est avant tout existence, c'est-à-dire mouvement vers le dehors, "pro-jet". Existence, et non essence: l'homme n'est pas ce qu'il est (comme le caillou est caillou, de part en part), il $a$ à être ce qu'il est parce qu'il ne l'est jamais d'emblée, ni de façon définitive, sauf dans la mort. L'homme est quelqu'un qui s'invente à tout moment, il est une autocréation continue; ce qui, d'ailleurs, ne l'empêche pas de nier sa liberté, de se 
comporter comme un objet, une passivité. Cette forme d'inertie, de négation de soi, qui conduit à toutes les lâchetés, Sartre l'appelle la mauvaise foi. La mauvaise foi est la comédie qu'on se joue à soimême et par laquelle on justifie toutes ses démissions, on récuse ses responsabilités, notamment à l'égard de la liberté, partout où elle s'affirme.

Mathieu apparaît dès l'abord comme un héros sartrien - ce n'est pas un hasard si Bruno et Danielle, ses amis, montent Les Mouches, où Sartre développe très clairement la problématique de la liberté et présente un héros solitaire forcé d'assumer ses choix, face à une société qui s'est enfermée dans un deuil et un devoir hypocrites. Françoise Loranger connaît bien l'œuvre de Sartre et elle en reprend en bonne partie la tonalité, c'est-à-dire l'aspect contestataire (on parle ici de contestation à un niveau fondamental, ontologique). Décaper la société, faire apparaître la vérité sous les mensonges, tel est le projet de Matbieu. Il s'agit d'aller vers la profondeur et, pour celà, de dissiper les mirages de la surface.

Mathieu étant un être lucide et écorché, incapable de vivre en société, son moi est d'une autre envergure que celui des gens qu'il côtoie. Les êtres autour de lui, tels du moins qu'il les perçoit, ont des contours fermement dessinés, et on pourrait dire que leur existence s'emploie à incarner une essence - Lucienne est l'épouse trahie mais digne, qui surmonte bravement son épreuve; Eugénie Beaulieu est la maîtresse de maison accomplie, la femme et la mère attentive; Nicole est l'enfant gâtée, sympathique, sans orgueil et sans talent, qui arrive à ses fins grâce à sa situation de fortune, etc. Loin d'être libres, ils sont complètement dépendants d'une identité - un caractère, un tempérament, une fonction sociale. Leur moi est simple et défini à la surface de leur être, du reste ils ne sont que surface. D'où leur transparence. Mathieu les connaît complètement. Sa mère, par exemple: "Appuyée sur une canne dont elle prétendait ne pouvoir se passer, Lucienne Normand marchait avec peine et lenteur" (p. 11). Dès cette présentation (à travers les yeux de Mathieu), le côté misérabiliste et faux de cette femme est suggéré, et la suite ne fera que le confirmer. Et voici maintenant son amie d'enfance, Eugénie Beaulieu, marraine de Mathieu: "Corps douillet, joues pleines et douces, regard agréablement vide, bouche molle et doigts potelés, toute la personne d'Eugénie Beaulieu témoignait de son existence privilégiée. / "Une bonne grosse oie bien nourrie, bien dodue" (p. 12). "Le moi d'une bourgeoise à l'aise dans l'existence, sans problème qui vaille, d'un côté; et celui d'une déclassée, hantée par un drame impossible à liquider, de l'autre. Le moi polochon et le moi ulcère. 
Mathieu, lui, est bien plus que cela, il est un moi à double ou triple fond. À la surface, il est un jeune homme pauvre et réservé, qui se retranche derrière ses verres fumés, coupant ainsi la communication avec les autres. Il s'abstrait du circuit des regards, des échanges, pour devenir un observateur solitaire, quelqu'un qui a un point de vue sur le circuit, sur les échanges, et qui domine donc la scène où s'établissent les premiers contacts. Il se situe au second degré. De la sorte, il compense pour la médiocrité de cela qui le définit au premier degré, par exemple sa situation de "parasite " au milieu des "héritiers" (p. 21). "Si tout cela pouvait me laisser indifférent! Si je parvenais au moins à m'habituer à toutes les humiliations de cette sale petite vie! Faire comme les autres, m'envelopper dans la routine des jours, dans la monotonie des gestes quotidiens" (p. 22). Mathieu aimerait bien, en somme, être comme les autres, vide, sot et aimable, se complaire dans le monde des essences, de ce qui a un nom, de ce qui permet de fonctionner. Mais à ce niveau premier, il est en déficit et rejeté vers une autre position, douloureuse, qui le met en dehors et au-dessus. C'est la position de la conscience, qui est toujours d'abord conscience d'un manque, d'une condamnation à exister plutôt qu'à être (comme le bourgeois ou le caillou). "Si je pouvais arriver à ne plus être conscient de tout ce qui m'arrive, à ne plus souffrir de la présence des autres! Pourquoi faut-il que les actes les plus familiers m'apparaissent sans cesse comme des événements nouveaux, jamais vus, jamais sentis (ibid.)!" En somme, spectateur des autres et de soi-même, du "premier degré ", Mathieu voit sous l'apparence habituelle, sous l'essence, ce mouvement de l'existence par lequel on se conforme à l'essence et nie sa liberté. Ce qu'il y a, pour Mathieu, de toujours neuf dans le spectacle des êtres qui l'entourent, c'est la fraîcheur du mensonge, la négation toujours reprise de l'existence (et de la conscience). Mathieu, certes, ne s'estime pas supérieur pour autant. Il souffre trop de sa lucidité pour ne pas souhaiter avoir comme lot, lui aussi, la bêtise ordinaire. La liberté, la conscience, c'est une malédiction: "Je suis au milieu d'eux comme un microbe parmi les microbes. La seule différence entre eux et moi, c'est que je le sais et qu'ils l'ignorent, c'est que j'en meurs et qu'ils en vivent" (ibid.). Les gens trouvent, dans leur petitesse, ce qu'il faut pour les rassurer et pour triompher des difficultés de la vie. Mathieu connaît plutôt l'écrasante disproportion entre des aspirations indéfinies, indéfinissables, qui sont celles mêmes de l'existence, et les menues satisfactions qu'apporte le fait de se conformer aux essences, aux principes, aux bienséances, aux diverses retombées de cette transcendance que l'humanité a inventée pour conjurer l'horreur de la mort. Pour vivre, il faut ignorer l'existence. La connaître, c'est connaitre ce par quoi on ne coïncide jamais avec soi, 
on est toujours déporté au-delà — bref, l'existence est ce qui contient en soi la mort.

\section{Le troisième dessous}

Mathieu a donc deux fonds: il est le "microbe" (premier niveau), et il est celui qui se voit être le microbe (deuxième niveau). Mais il est aussi celui qui s'admoneste, et cela, dès les premières lignes du livre:

Hésitant sur l'attitude à prendre, il opta pour la désinvolture et alla ranger le volume dans la bibliothèque. Mais il mentait aux autres mieux qu'à lui-même. (p. 11)

Mathieu dissimule à sa-mère et à sa marraine, qui viennent vers lui, son occupation: il lit de la poésie. Au premier niveau, il y a les deux femmes qui font intrusion dans l'univers du jeune homme et ce dernier, qui se cache d'elles ou, comme dit l'auteur, qui leur ment. Au deuxième niveau, il y a ce que le jeune homme veut protéger: son intériorité. La sensibilité à la poésie pourrait passer pour une faiblesse. Dans l'univers des adultes, où l'on n'existe que pour autrui, où l'on est complètement absorbé par son rôle social, la manifestation du poursoi est une discordance. Il ne faut pas exister pour soi mais se laisser déterminer par les bienséances. Il faut mentir, et c'est ce que fait Mathieu en annulant tout signe d'intériorité et de sensibilité. Mais voilà qu'une voix l'apostrophe: "- Collégien! Pourquoi te caches-tu d'aimer la poésie?"(ibid.)

Appelons contre-moi cet énonciateur étrange, dont les semonces viennent périodiquement contester les pensées ou les attitudes du héros, de façon toujours énergique, souvent à l'impératif ou sous forme de jugements péremptoires. Le contre-moi, ici, discute la discordance entre le pour-soi et le pour-autrui qui sont les deux dimensions du $m o i$, et on peut penser que tel sera son rôle, de façon générale de là le nom de contre-moi que je lui donne. Ses interventions s'appliquent essentiellement au discours de l'intériorité (moi profond), mais en général pour discuter la pertinence d'une attitude à l'égard d'autrui. Le côté abrupt des interventions, leur clarté qui en fait la voix même de l'autorité morale, rapproche ce contre-moi du surmoi des psychanalystes. On ne peut pas, cependant, les assimiler complètement. Le surmoi résulte de l'introjection de figures parentales, et il comporte notamment la norme sociale, médiatisée par ces figures. De plus, le surmoi agit en collaboration avec le ça et incarne la voix du devoir, un devoir qui se passe bien de justifications. Or, la voix intérieure de Mathieu, la voix du contre-moi, est sans doute impérieuse, morale, mais elle est aussi raisonnable, et elle est pleinement l'affaire de la 
conscience. Dans la réalité, personne ne s'apostrophe soi-même comme le fait Mathieu (ou comme quelqu'un le fait en Mathieu), de façon aussi claire et décidée. Cette particularité psychologique peu vraisemblable cherche simplement à accréditer la fiction d'un surmoi conscient, qui n'est pas sans rapport avec le fait que Mathieu, malgré toutes les raisons qu'il a d'être névrosé, en viendra un jour à liquider véritablement sa névrose. Ce qui, là aussi, peut sembler psychologiquement invraisemblable - ce n'est pas pour rien que Freud, au fil des ans, parlera de plus en plus d'analyse interminable. Le contre-moi de Mathieu, en somme, relève de la conscience et la santé mentale du personnage sera finalement régie par la raison, ce qui n'exclut pas l'existence d'un inconscient et de la névrose, mais suppose que la concience et la raison sont supérieures aux forces ténébreuses de l'être. Bref, il y a chez Loranger un optimisme remarquable, et c'est un autre aspect qui permet de la rapprocher de Sartre, à condition de faire les nuances qui s'imposent. On peut dire qu'il y a, chez Sartre, un optimisme théorique, puisque pour lui l'homme est libre et triomphe, ou peut triompher, des déterminismes psychologiques et sociaux. Sartre, dans les années trente et quarante, condamne la psychanalyse qui fait de l'homme le jouet de ses pulsions, et le marxisme qui dissout l'individu dans sa classe sociale. Par contre, en pratique, l'homme s'avère être de façon très générale le complice des oppressions et la victime consentante de sa mauvaise foi. L'homme est libre, mais il s'emploie constamment à nier sa liberté. De sorte que les récits de fiction de Sartre, ou ses pièces de théâtre, n'ont jamais de fin heureuse et s'emploient généralement à mọontrer l'enfer où vit chacun, du fait de sa mauvaise foi.

\section{Métaphysique du soi}

Mais pour Sartre - et on le lui a beaucoup reproché - l'inconscient n'est pas une fatalité, un déterminisme au sens où l'ont conçu les psychanalystes; l'homme a accès aux profondeurs de son moi puisque c'est la conscience libre qui se constitue elle-même à tous ses niveaux, et il n'y a donc pas de part réservée qui serait celle d'un autre, en soi, d'une autre scène. Chez Loranger, cette voix intérieure faisant office de surmoi, mais pleinement raisonnable, est l'expression de tout l'être et par conséquent, aussi, des pulsions, comme si une continuité existait entre le conscient et l'inconscient. Lisons le passage, long mais plein d'enseignements, où l'auteur décrit avec la plus grande précision ce que j'ai appelé le contre-moi en raison de la fonction critique qu'il assume longtemps et qui finit par se manifester en consonance avec le moi lorsque celui-ci triomphe enfin de ses mauvais instincts : 
Évidemment, il ne pouvait être question de vivre dans un paroxysme perpétuel, mais ne pouvait-il au moins espérer une espèce de sérénité confinant au bonheur, une paix profonde qui le mettrait à l'abri du désespoir auquel son éducation l'avait poussé? Comment, comment, comment atteindre cette sérénité?

Cherche, cherche en toi. C'est en toi qu'il faut regarder! Tu le sais, tu l'as toujours su.

Cette voix qui jaillissait par moments, secrète, intime, au plus profond de son être, en savait-elle plus long, sur le bonheur, que sa raison? Sur son bonheur?.. S'il avait toujours suivi ses avertissements au lieu de les repousser, serait-il aujourd'hui plus heureux? Ne semblait-elle pas provenir du meilleur de lui-même, de la partie la plus vraie; la plus pure, la seule qui comptât: celle qui doit dominer, diriger, unifier? Cette voix n'était-elle pas dictée tantôt par une intuition profonde qui savait, avant son intelligence et mieux qu'elle ce qui lui convenait essentiellement, tantôt par une conscience immanente qui le guidait vers le bien, un bien qui ne pouvait être que source de joie? Ne procédaient-elles pas l'une et l'autre, intuition et conscience, d'un désir, d'une volonté d'unification? Ne tendaient-elles pas également à coordonner les meilleurs éléments de l'être en vue de former une entité? En suivant à la lettre leurs ordres intimes, si pénibles soient-ils parfois, n'arriverait-il pas enfin à l'épanouissement total de toutes ses facultés, c'est-à-dire à la joie? (p. 249-250)

De ce passage important qui expose toute une conception métaphysique de l'être humain et de son aspiration au bonheur, je retiendrai un certain nombre de points, rattachés ou non à la voix intérieure elle-même, qui en est le motif principal.

D'abord, il est question de "ce désespoir auquel son éducation l'avait poussé". On pourrait penser à une forme de rousseauisme: l'homme naît naturellement heureux, mais son éducation le rendrait malheureux. Le désespoir ne résulte pas de dispositions naturelles mais culturelles (socialés). C'est Lucienne qui a élevé son fils pour le malheur, cherchant ainsi à se venger de l'homme qui l'a humiliée, abandonnée. Mathieu serait l'instrument, depuis son plus jeune âge, d'une volonté mauvaise et antérieure à lui, mais il lui suffirait de prendre conscience de ces enjeux particuliers pour échapper enfin au malheur qui l'accable. De même que, pour Sartre et Simone de Beauvoir, il n'y a pas de nature humaine mais seulement une condition humaine, c'est-à-dire le fait d'une liberté qui a à se choisir dans dẹs situations données, de même Mathieu peut-il, par une prise de conscience décisive, se dépêtrer du long esclavage psychologique auquel l'a contraint sa mère.

Françoise Loranger ne suit quand même pas Sartre jusqu'au point de nier la nature humaine, même si elle croit elle aussi au condition- 
nement culturel (par exemple, celui de l'éduçation, du milieu familial ou scolaire). Une situation répétée a sans doute fait de Mathieu l'être hargneux et malheureux qu'il est, mais c'est en puisant dans le trésor de sa nature profonde qu'il parviendra à s'en sortir. L'homme n'est pas une pure liberté qui se détermine en situation, il est en continuité avec les forces vives qui composent la réalité humaine, et en particulier les ressources physiques, le corps. Entre la santé physique et la santé psychologique, il y a une relation très étroite que Sartre jugeait fort discutable (Simone de Beauvoir, elle, y souscrirait plus aisément) et qui s'avère, pour Françoise Loranger, déterminante. Comme est déterminante, poúr elle, l'aspiration de chacun au bonheur: thème inexistant chez Sartre, mais très important, là encore, chez Simone de Beauvoir qui donne le rare exemple d'un existentialisme athée (donc "désespéré") en même temps empreint d'optimisme réel et de goût pour la joie et pour tous les bonheurs corporels. Françoise Loranger, dans la mesure où elle est proche des existentialistes, est certainement plus proche de Simone de Beauvoir que de Sartre.

Où puiser la sérénité? "Cherche, cherche en toi ", répond la voix, dont les accents rappellent beaucoup ceux du personnalisme chrétien. Le bonheur, athée ou non, ne réside donc pas dans la négation du moi, dans une pure ouverture à l'extérieur - au monde, aux autres qui est l'attitude des adultes courants, des bien-pensants, de ceux qu'on pourrait appeler les hommes ou les êtres "de là ${ }^{7}$. Il faut s'assumer soi-même pour connaître la joie d'exister. Mais alors, il importe de dépasser cette couche de malheur qui forme le plus souvent l'intériorité des êtres tournés vers eux-mêmes (l'“homme d'ici"). Au fond du malheur, qui d'ailleurs se développe à cause d'une mauvaise relation avec les autres (c'est son éducation qui voue Mathieu à la névrose); il y a quelque chose de soi qui est inaliénable, positif et qu'on sait être là depuis toujours. L'homme est conscient des ressources de joie qu'il possède en lui, même s'il les nie avec vigueur comme le fait Mathieu pendant de nombreuses années de sa vie. Le savoir de la joie en soi, c'est précisément cette conscience intégrale qui, par un processus semblable à la mauvaise foi chez Sartre, se nie elle-même la plupart du temps et pourtant s'affirme parfois avec netteté à travers la voix intérieure. Chez Sartre, il n'y a rien cependant, en dehors de la liberté qui s'affirme ou se nie; aucune voix distincte nè fait entendre la vérité sur soi, à partir d'une nature juste, droite, correspondant à ce qu'il y a de "meilleur" en soi, à la "partie la plus vraie, la plus pure, la seule qui comptât". Cette partie de soi n'est pas le moi au sens de la monade, de l'individualité close sur elle-même, lorsque Mathieu se vit pour soi dans les heures de désespoir. Il est le moi communiquant, par 
en-dessous, avec l'être, grâce au dynamisme et à l'équilibre des forces naturelles, physiques. C'est un moi naturé et naturant, aux antipodes des abstractions dont se repait volontiers le moi narcissique, souvent réfugié dans une intelligence stérile et coupée du corps, de la nature, de toute réalité.

La voix intérieure n'est donc pas celle du moi fermé sur lui-même, mais de ce qu'on pourrait appeler un Soi; ouvert à la nature humaine et au..bonheur qui en découle quand on ne s'en est pas détourné. C'est la voix, pourrait-on dire, du sujet, opposée au discours égoïste de l'individu, selon la distinction proposée par Alain Renaut dans $L$ 'Ére de l'individu ${ }^{8}$. Or, cette voix du sujet n'est pas sans rapport, chez Loranger du moins, avec un certain sens de la hiérarchie et de la directivité, bref avec la figure paternelle et ce qu'elle implique d'autorité. (On sait que Louis Dumont associe la société traditionnelle à la hiérarchie, et la société moderne - "démocratique" - à l'égalité.) Tout se passe comme si, chez Mathieu, le moi était négatif, fermé et anarchique, alors que le contre-moi, ou sujet, se montrait positif, ouvert au monde et directif; il est, de Mathieu, la partie "qui doit dominer, diriger, unifier ". Le moi, lui, ne peut que détruire ou du moins subvertir, contester, car il est aux prises avec l'arbitraire social (ou religieux) qui lui est imposé de l'extérieur, comme une dérisoire transcendance. C'est par la raison que le moi mène son combat contre l'arbitraire; la raison qui analyse, dissèque, mais qui est prisonnière de ce travail d'analyse. L'action - du moins, une certaine action qui s'apparente à l'agitation - reste le privilège des autres, de la société. Mathieu, par exemple, se laisse mener par sa mère; avec Nicole et ses cousins, il se trouve relégué au second plan et voué à la passivité. L'être d'ici ne peut guère jouer d'autre rôle que celui de témoin. Il observe tout en se retranchant derrière ses lunettes noires: le témoin voit mais n'est pas vu, du moins n'est pas vu dans son activité d'être qui voit.

Mathieu tient beaucoup à sa raison, puisqu'elle lui permet d'adopter un point de vie supérieur sur les autres, toujours perçus comme des agresseurs potentiels - l'autre par excellence étant sa mère, qui le brutalise psychologiquement encore à vingt-cinq ans ${ }^{9}$, après l'avoir brutalisé physiquement jusqu'à un âge relativement avancé. La raison est une arme contre la violence d'autrui. Mais elle n'est d'aucun secours pour conduire Mathieu au bonheur, qui relève, non pas de la relation à autrui (les autres sont des ennemis), ni du moi (microbe parmi les microbes), mais d'une espèce de conscience profonde èt oblitérée au sein de laquelle, par exemple, peut se forger l'élan amoureux, dans lequel l'être est pleinement Moi tout en étant pleinement 
tourné vers l'autre. L'amour de Mathieu pour Danielle part de cette même région profonde que la voix qui l'admoneste et qui, par là, prétend le diriger. L'aspect impérieux de la voix intérieure (qui s'adresse à Mathieu à l'impératif: "Cherche, cherche en toi"), cette conscience paternelle qui montre la direction à suivre, c'est aussi ce qui permet le dépassement vers l'autre, vers la femme. L'unification de soi est le prélude à l'union amoureuse.

Qu'est-ce que l'unification de soi sinon le comblement de la béance créée par l'agression d'autrui ("l'éducation", dans le cas de Mathieu), et la constitution d'un moi séparé, origine de tous les tourments?

Pour suturer la blessure profonde et rétablir l'harmonie entre le $m o i$ et le monde, Mathieu doit être mis en présence de ce qui l'habite au plus profond de lui-même et qui détient le secret de la joie, c'est-àdire du plein épanouissement de soi. Ce qui l'habite donc, et qui d'ailleurs se signale à lui par la voix intérieure (celle du contre-moi), c'est le Père. Non pas le père biographique, cet être lamentable, autrefois beau et maintenant réduit à l'état de loque humaine; mais le père tel que l'enfant, puis l'adulte, le portent en eux, le père essentiel, directif mais non écrasant, sorte d'idéal du moi qui représente ce qu'Alain Renaut appelle la transcendance de l'immanence. La découverte du père, qui ne fait qu'un avec celle de la joie et qui est aussi la découverte de l'instance qui s'exprime par lá voix impérieuse, se fait en trois étapes précises, associées aux noms du Mont-Royal, de la Toccate et Fugue en ré mineur (de Jean-Sébastien Bach) et d'Émile Rochat: la nature, la culture et la présence humaine.

(L'étude se poursuit par l'examen de ces trois motifs, puis par l'analyse de personnages proches de Mathieu grâce à leur sens de l'intériorité, Étienne et Danielle. Elle montre ensuite comment l'auteur surmonte la tentation religieuse propre à sa génération.)

1. Extrait d'une étude en préparation sur les romans du moi dans le Québec des années cinquante.

2. Michel Tremblay, Un ange cornu avec des ailes de tôle, Marseille, Actes Sud, 1994, p. 159.

3. Ibid.

4. On relira, sur ce grand roman réaliste et sur Bonbeur d'occasion qui en est l'une des rares manifestations au Québec, et certainement l'une des plus importantes, les pages suggestives de Gilles Marcotte dans son Roman à l'imparfait, Montréal, La Presse, 1978.

5. Nous nous référons à la dernière édition, Montréal, Boréal, coll. "Compact ", 1990, 404 p. L'édition originale, au Cercle du livre de France, est de 1949.

6. Voir Louis Dumont, Essais sur l'individualisme, Paris, Seuil, 1983, 272 p.;'et Alain Renaut, L'Ére de l'individu, Paris, Gallimard, coll. "Bibliothèque des idées", 1989. 
7. L'expression est d'Ernest Gagnon qui, dans L'Homme d'ici (Montréal, HMH, coll. "Constantes", 1963; l'édition originale, à l'Institut littéraire de Québec, parut en 1952), oppose l'homme de là, extraverti, orienté vers l'action, les certitudes et les principes, à l'homme d'ici, introverti, méditatif, confiné dans sa vie intérieure.

8. Alain Renaut, op. cit. Le sujet est en relation avec les autres sujets, qui sont ses égaux (par exemple, chez Descartes), alors que l'individu est une monade "sans porte ni fenêtre " (Leibniz).

9. Vingt-huit ans, lit-on à la page 217 (édition Boréal). Information démentie à la page 264 . 